Nature Reviews Genetics | AOP, published online 12 April 2011; doi:10.1038/nrg2991

$\Rightarrow$ DISEASE EPIGENOMICS

\title{
A smoking gun
}

A study using genome-wide epigenetic profiling has shown that heavy smoking causes reduced levels of DNA methylation in a gene that encodes a drug target for cardiovascular disease.

Methodological limitations have meant that most studies of the potential links between DNA methylation and disease have so far focused on those $\mathrm{CpG}$ (the most common sites of DNA methylation in humans) for which there is pre-existing reason to suspect a disease connection. Breitling and colleagues used an array-based platform for genome-wide DNA methylation profiling to study the effects of cigarette smoking, free of any a priori hypothesis about which positions might be affected. DNA methylation was measured at $27,578 \mathrm{CpG}$ in promoters across the genome in peripheral blood from 177 people, including heavy smokers, former heavy smokers and those who had never smoked.

The authors identified one site in the gene coagulation factor II receptor-like 3 (F2RL3) - that showed genome-wide significance for reduced DNA methylation in heavy smokers. This association was replicated in an independent sample of 328 people. Excitingly, despite the fact that it had not previously been implicated in the effects of smoking, F2RL3 encodes a protein that has functions which are relevant to cardiovascular disease, and is in fact a potential drug target for that condition.

Although the functional impact of this difference in DNA methylation remains to be explored, this study illustrates the potential of epigenomics to provide insights into mechanisms of disease, and to suggest new avenues for clinical intervention.

Louisa Flintoft

ORIGINAL RESEARCH PAPER Breitling, L. P. et al. Tobacco-smoking-related differential DNA methylation: $27 \mathrm{~K}$ discovery and replication.

Am.J. Hum. Genet. 31 Mar 2011 (doi:10.1016/ j.ajhg.2011.03.003) 